\title{
Quistes mesoteliales benignos con posible origen en las trompas de Falopio resecados por laparotomía
}

\author{
Benign mesothelial cysts with possible origin in the Fallopian tubes resected \\ by laparotomy
}

Jorge Alberto Bernal-Mesa' $\mathbb{D}$, Juan Jacobo Del Valle-Saavedra² $\mathbb{D}$, Federico López-Uribe $\mathbb{D}^{\mathbb{D}}$

1 Médico, especialista en Cirugía general, subespecialista en Cirugía oncológica y Laparoscopia avanzada; docente, Universidad CES, Medellín, Colombia.

2 Médico, residente de Dermatología, Universidad CES, Medellín, Colombia.

3 Médico, residente de Cirugía general, Universidad CES, Medellín, Colombia.

\section{Resumen}

Introducción. Los quistes mesoteliales benignos son una entidad poco frecuente, que ocurren especialmente en mujeres en edad reproductiva. Se deben a una proliferación anómala del mesotelio que puede ser originada en varias estructuras intraabdominales e inicialmente es asintomática.

Caso clínico. Se presenta el caso de una mujer de 20 años con un cuadro sugestivo de neoplasia maligna, en quien se confirmó el diagnóstico de quiste mesotelial originado en la trompa de Falopio derecha. Se realizó tratamiento quirúrgico exitoso.

Discusión. A pesar de la estrecha relación de esta entidad con el útero y los ovarios, no se encuentran reportes de quistes mesoteliales originados a partir de las trompas de Falopio. El tratamiento de los quistes mesoteliales es quirúrgico y en el caso de esta paciente se hizo con la finalidad de mejorar los síntomas y erradicar la enfermedad. Las decisiones fueron tomadas basadas en la opinión de expertos en oncología en una junta de tumores gastrointestinales.

Conclusiones. Los quistes mesoteliales son neoplasias de baja incidencia, pero se deben tener en cuenta como diagnóstico diferencial de los tumores abdominales. Se presenta el caso de una paciente tratada de forma quirúrgica con resultados exitosos y seguimiento a un año sin recurrencias.

Palabras clave: neoplasias; mesotelioma quístico; trompas de Falopio; laparotomía; histología.

Fecha de recibido: 31/07/2020 - Fecha de aceptación: 22/11/2020 - Fecha de publicación en línea: 23/07/2021 Autor de correspondencia: Jorge Alberto Bernal-Mesa, carrera 27 D \# 27 sur 98 casa 117, Envigado, Colombia. Teléfono: 3104078299. Dirección electrónica: laparoscopiaavanzada@gmail.com

Citar como: Bernal-Mesa JA, Del Valle-Saavedra JJ, López-Uribe F. Quistes mesoteliales benignos con posible origen en trompas de Falopio resecados por laparotomía. Rev Colomb Cir. 2021;36:726-31. https://doi.org/10.30944/20117582.738

Este es un artículo de acceso abierto bajo una Licencia Creative Commons - BY-NC-ND https://creativecommons.org/licenses/by-ncnd/4.0/deed.es 


\begin{abstract}
Introduction. Benign mesothelial cysts (BMC) are a rare entity that occurs especially in women of reproductive age. It is due to an abnormal proliferation of the mesothelium that can originate from various intra-abdominal structures and it is initially asymptomatic.
\end{abstract}

Clinical case. This is a case of a 20 -year-old woman with a suspicious malignant neoplasia, in whom the diagnosis of a mesothelial cyst originating in the right Fallopian tube was confirmed. Successful surgical treatment was achieved.

Discussion. Despite the close relationship of this entity with the uterus and ovaries, there are no reports of mesothelial cysts originating from the Fallopian tubes. The treatment of mesothelial cysts is surgical and in the case of this patient it was performed in order to improve the symptoms and eradicate the disease. The decisions were taken based on the oncologists' expert opinion of the gastrointestinal tumor board.

Conclusions. Mesothelial cysts are neoplasms of low incidence, but they should be considered as a differential diagnosis of abdominal tumors. The case of a patient treated surgically with successful results and a one-year follow-up without recurrences is presented.

Keywords: neoplasms; cystic mesothelioma; Fallopian tube; laparotomy; histology.

\section{Introducción}

Los quistes mesoteliales benignos (QMB), conocidos también como mesoteliomas peritoneales multiquísticos o quistes de inclusión peritoneal, son neoplasias originadas en el peritoneo, de muy baja incidencia y que tienden a aparecer en la edad reproductiva, alrededor de los 36 años en las mujeres y a una mayor edad en los hombres, en quienes es menos común ${ }^{1,2}$. Estas neoplasias son el resultado de la proliferación celular entre las células epiteliales y mesenquimales del mesotelio, una de las capas de recubrimiento visceral del peritoneo ${ }^{3}$.

Se presenta el caso de una paciente joven con un quiste mesotelial benigno originado en la trompa de Falopio derecha, diagnóstico confirmado por la resonancia nuclear magnética, los hallazgos quirúrgicos y el estudio histopatológico.

\section{Caso Clínico}

Paciente femenina de 20 años, natural de Medellín, Colombia, con antecedente personal de pólipo uterino. Consultó por un cuadro clínico de seis meses de evolución de distensión y dolor abdominal progresivo, mal apetito y una pérdida aproximada de $7 \mathrm{~kg}$ de peso. Posteriormente presentó dolor asociado al decúbito lateral y disnea. Al examen físico se encontró un abdomen blando, sin masas y con ausencia de adenopatías en las cadenas ganglionares.

Se realizó una resonancia magnética de abdomen y pelvis que demostró la ocupación de todos los recesos peritoneales con líquido complejo de diferentes captaciones y septos multiloculados, la ocupación del espacio subfrénico derecho debido al desplazamiento tanto del hígado en sentido superior, como de las asas intestinales incluyendo el colon derecho en sentido central y en sentido posterior rechazando el colon descendente. No había compromiso retroperitoneal. Se encontró además una lesión quística dominante adyacente al pedículo ovárico derecho sin conformarse propiamente una masa ovárica, lo que hizo sospechar el origen en la trompa. Debido a la presentación clínica y a los resultados de la resonancia, se sospechó un pseudomixoma peritoneal de probable origen apendicular (Figuras 1-3).

Se realizo una laparoscopia diagnóstica con tres trócares en la línea media y luego del neumoperitoneo se exploró la cavidad peritoneal encontrando múltiples lesiones quísticas de contenido seroso y hemorrágico sobre el intestino y el omento, que estaban comunicados con la pelvis. Se tomaron muestras del líquido peritoneal y de los quistes peritoneales para estudio citológico y patológico. 


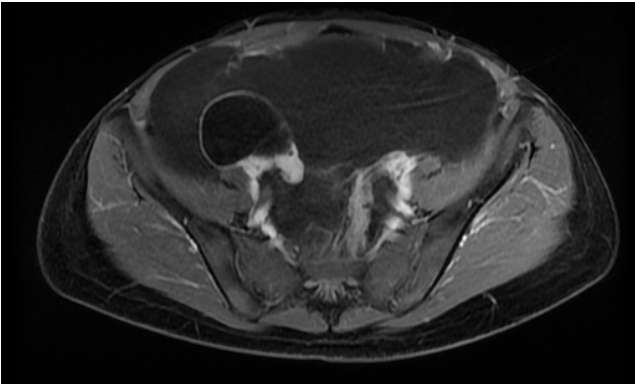

Figura 1. Resonancia magnética abdominal en corte T1 con supresión grasa post contraste (corte axial). La trompa derecha está adherida a la lesión quística pura, sin contenido proteínico ni sanguinolento, que aparentemente corresponde a la lesión quística inicial de la paciente.

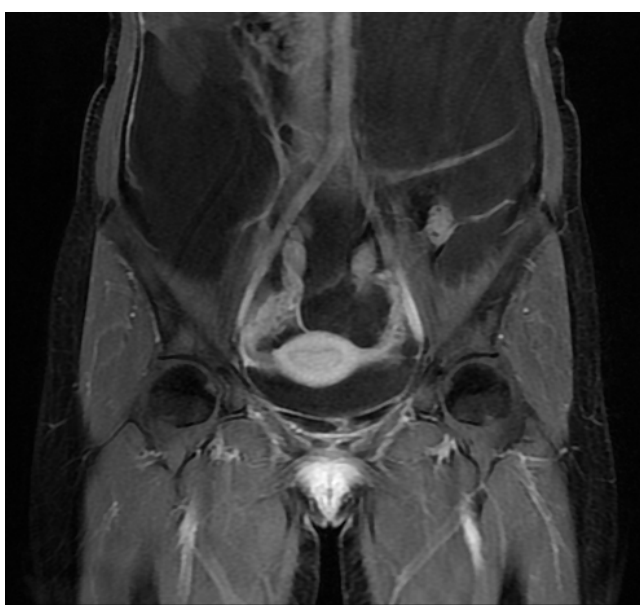

Figura 2. Resonancia magnética abdominal en corte T1 con supresión grasa post contraste (corte coronal). Se observa la primera lesión quística en la trompa derecha.

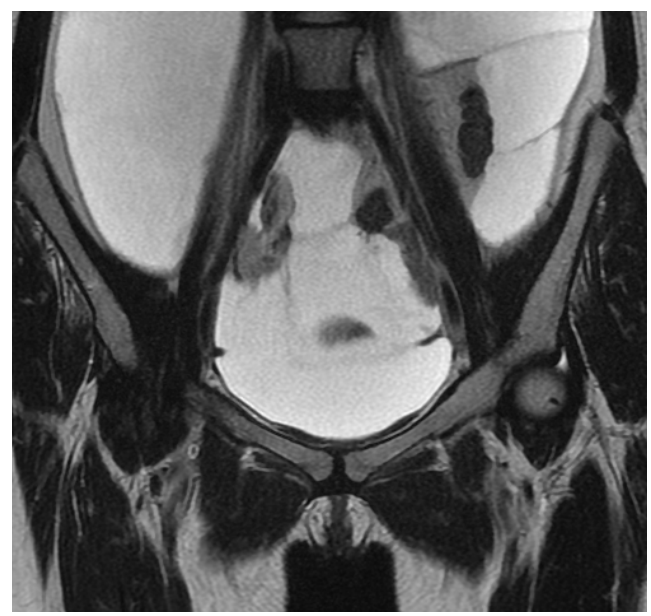

Figura 3. Resonancia magnética abdominal en corte coronal T2 con supresión grasa. Se observa el origen del primer quiste en la trompa de Falopio derecha.
El reporte de la biopsia del quiste informó una pared compuesta por tejido conectivo laxo, tapizada por células cuboidales y planas dispuestas en monocapa, sin displasia. Además, se amplió el estudio con marcadores de inmunohistoquímica: citoqueratinas CK7 y CK20, calretinina, CD34, p53, WT1 y D2-40, los cuales descartaron malignidad y favorecieron el diagnóstico de QMB. En la citología del líquido peritoneal se encontró un fondo de aspecto proteínico, eosinófilos, células inflamatorias polimorfonucleares neutrófilos y algunas células mesoteliales, apoyando el diagnóstico de lesión benigna.

Teniendo en cuenta el resultado de patología con marcadores específicos, se presentó el caso en un comité de tumores gastrointestinales, y se decidió realizar la resección de los quistes mesoteliales mediante laparotomía mediana supra e infraumbilical, debido al riesgo de ruptura de los quistes en caso de manejo laparoscópico. Durante el procedimiento se identificaron múltiples quistes peritoneales, algunos con contenido seroso y otros con contenido hemorrágico, adheridos entre si por cordones fibrosos, algunos adheridos a la trompa de Falopio derecha. Se realizó ligadura de los pedículos vasculares y sección de las adherencias a la trompa de Falopio, sin presentar complicaciones durante el procedimiento (Figuras 4-6).

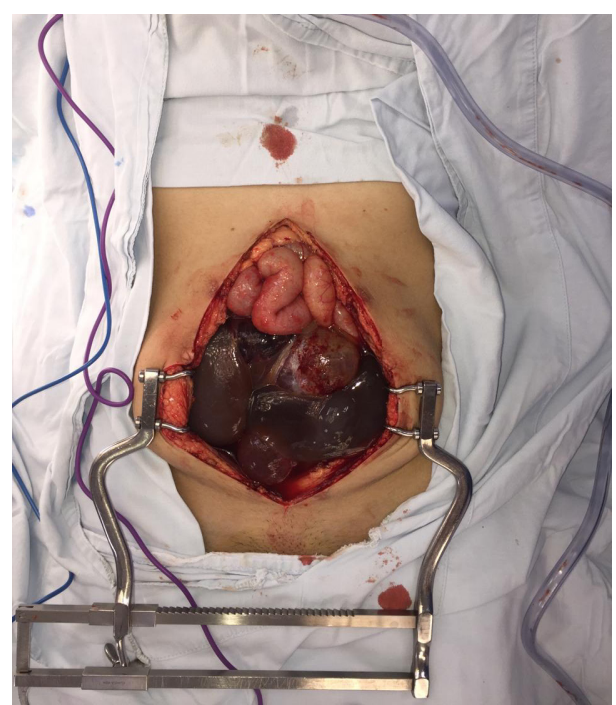

Figura 4. Laparotomía mediana supra e infraumbilical. Se observa la exposición de las asas de intestino delgado rechazadas en sentido cefálico por los quistes mesoteliales. 


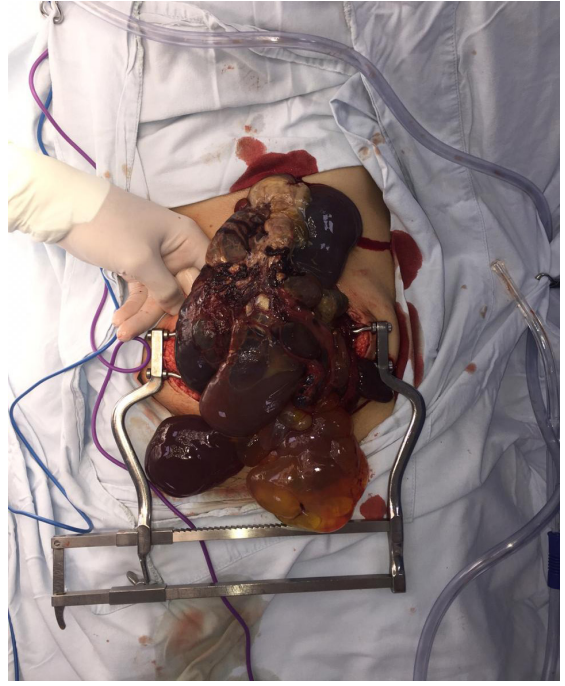

Figura 5. Resección de quistes mediante laparotomía, se visualizan múltiples quistes con diferente contenido, hemorrágico y seroso.

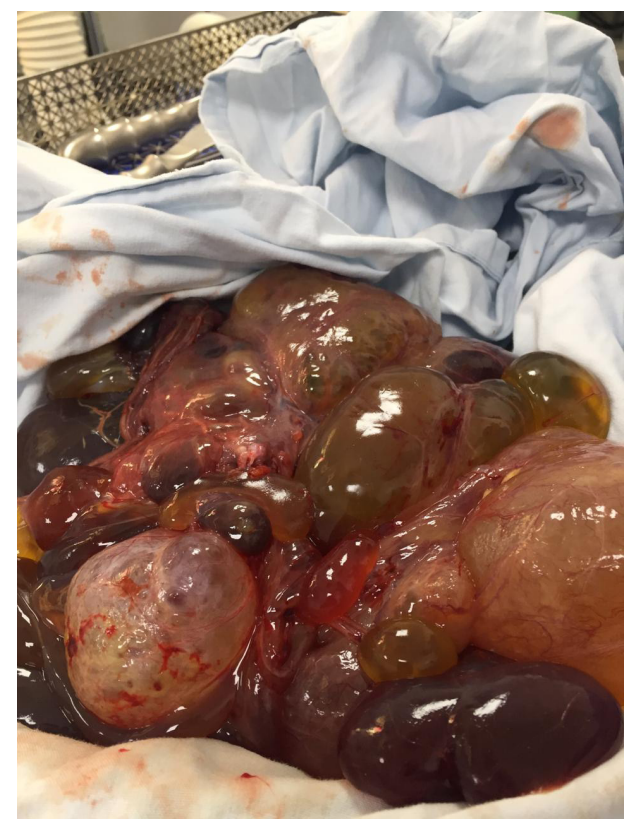

Figura 6. Detalle de los quistes resecados. Se observan las paredes y sus componentes.

\section{Discusión}

Los QMB son entidades infrecuentes con patogénesis incierta, y por ser más común en mujeres que en hombres, se ha relacionado con causas hormonales ${ }^{1,4}$, pero también se han implicado factores ambientales e incluso el trauma físico ${ }^{3}$. Algunos autores mencionan una proliferación incrementada de las células mesoteliales que sufren un proceso de diferenciación celular, con células mononucleares que se adhieren a las capas mesoteliales generando los quistes. Se ha encontrado relación con la endometriosis, la enfermedad renal, cirugías abdominales previas y antecedente de consumo de alcohol y tabaquismo ${ }^{1,4}$.

Para establecer el diagnóstico es necesario contar con una historia clínica adecuada, estudios imagenológicos que confirmen la presencia de quistes y estudios histológicos que descarten otras patologías más frecuentes. La clínica se caracteriza por dolor abdominal crónico, sensibilidad y distensión abdominal, masas en la cavidad abdominal o en la pelvis y, en menor medida, puede haber pérdida de peso. Debido a los sitios de crecimiento de las lesiones, se puede encontrar sintomatología diversa como obstrucción intestinal o molestias de vías urinarias. Se debe tener en cuenta que varios pacientes pueden ser asintomáticos, lo cual puede retrasar el enfoque inicial y por consiguiente el diagnóstico oportuno ${ }^{1,4}$.

Entre las diferentes técnicas imagenológicas utilizadas como parte del enfoque, se describe la ecografía, la tomografía computarizada de abdomen y la resonancia magnética de abdomen. Según su origen, los quistes mesoteliales pueden detectarse inicialmente por ecografía de abdomen y pelvis, donde se observan múltiples quistes septados en relación anatómica con el útero y los anexos, generalmente con fluido anecoico. Imágenes como la tomografía computarizada o la resonancia se utilizan para definir la extensión de las lesiones y contribuyen en la evaluación de diagnósticos diferenciales, como los pseudomixomas peritoneales y otras neoplasias primarias viscerales ${ }^{5}$. En la tomografía de abdomen se reportan generalmente lesiones de baja densidad, multiquísticas y multiloculadas, de paredes delgadas que pueden estar inmersas en los tejidos blandos adyacentes, pero sin invasión a estos. En la resonancia abdominal se observan masas quísticas multiloculadas con paredes que pueden mostrar realce tenue con el medio de contraste, sin encontrar grasa dentro de los quistes ${ }^{6,7}$. 
En los estudios patológicos, las masas quísticas uniloculares o multiloculares se encuentran revestidas por una capa de células mesoteliales en patrones planos o cuboidales. Entre los marcadores de inmunohistoquímica, se encuentran positivos los marcadores de calretinina, citoqueratinas, vimentina y a veces marcadores hormonales, mientras que hay negatividad para los marcadores CD31, CD34 y factor del receptor de crecimiento vascular endotelial ${ }^{1,8}$.

Debido a lo inusual de esta patología, no existen guías clínicas que permitan un enfoque diagnóstico ni terapéutico especifico, se han propuesto diferentes tratamientos como la resección quirúrgica en bloque por medio de laparoscopia, que además puede tener un enfoque diagnóstico. También se ha descrito combinación de tratamientos, como escleroterapia, cirugía citorreductora y quimioterapia intraperitoneal hipertérmica, que puede proporcionar un mejor control de la enfermedad, disminuyendo la tasa de recaídas en comparación con el tratamiento quirúrgico único. La técnica y la forma de resección no se ha establecido y es a criterio del cirujano, de acuerdo con el contexto de la enfermedad, la disponibilidad de insumos y la experiencia con los diferentes abordajes $^{9-11}$.

Al realizar la laparoscopia diagnostica en la paciente presentada, se encontró que, además del gran volumen, los quistes tenían una pared delgada con alto riesgo de ruptura por la instrumentación laparoscópica, por lo que se decidió realizar la resección a través de una laparotomía mediana, para asegurar una disección completa de los quistes evitando su ruptura.

En vista de las altas tasas de recidiva de esta enfermedad, que llegan hasta un $50 \%$, se han estudiado otras opciones terapéuticas que incluyen el uso de tamoxifeno y agonistas de la hormona liberadora de gonadotropina ${ }^{11,12}$, con el fin de lograr la reducción del tamaño de los quistes, y de los síntomas, constituyéndose además en una alternativa terapéutica en caso de recurrencias. Diversos autores no recomiendan el uso de quimioterapia adyuvante ni de radioterapia para esta enfermedad ${ }^{4,11-13}$.

\section{Conclusiones}

Los quistes mesoteliales benignos son neoplasias de muy baja incidencia, más cuando son originados en las trompas de Falopio. Deben ser tenidos en cuenta como causa de tumores abdominales en aquellos pacientes en los cuales se sospeche un síndrome tumoral abdomino-pélvico con características clínicas e imagenológicas específicas.

No existen protocolos ni guías de manejo específicas para este tipo de tumor, por lo que un buen criterio médico, asociado a estudios radiológicos e histopatológicos adecuados, pueden orientar a un diagnóstico y enfoque terapéutico adecuado.

Después de un año de seguimiento, la paciente ha permanecido estable, con mejoría sintomática y sin recaídas tumorales.

\section{Cumplimiento de normas éticas}

Consentimiento informado: este artículo es la descripción retrospectiva de un caso clínico, por lo que no implicó riesgo alguno para la paciente, y se contó con el consentimiento quirúrgico informado por parte de ella.

Conflictos de interés: ninguno declarado por los autores.

Fuentes de financiación: Financiado con recursos propios de los autores.

\section{Contribución de los autores:}

Concepción y diseño del estudio: Jorge Alberto BernalMesa, Juan Jacobo Del Valle-Saavedra, Federico LópezUribe.

Adquisición de datos: Jorge Alberto Bernal-Mesa, Juan Jacobo Del Valle-Saavedra, Federico López-Uribe.

Análisis e interpretación de datos: Jorge Alberto BernalMesa, Juan Jacobo Del Valle-Saavedra, Federico LópezUribe.

Redacción del manuscrito: Jorge Alberto Bernal-Mesa, Juan Jacobo Del Valle-Saavedra, Federico López-Uribe.

Revisión crítica: Jorge Alberto Bernal-Mesa, Juan Jacobo Del Valle-Saavedra, Federico López-Uribe.

\section{Referencias}

1. Zhang C, Yu J, Luo M. Multicystic peritoneal mesothelioma: A short review. Curr Probl Cancer. 2017; 41:340-8. https://doi.org/10.1016/j.currproblcancer.2017.03.002 
2. Khurram MS, Shaikh H, Khan U, Edens J, Ibrar W, Hamza A, et al. Benign multicystic peritoneal mesothelioma: a rare condition in an uncommon gender. Case Rep Pathol. 2017;2017:1-4. https://doi.org/10.1155/2017/9752908

3. Myers DJ, Babiker HM. Benign mesothelioma. En: StatPearls. Treasure Island (FL): StatPearls Publishing. Disponible en: http://www.ncbi.nlm.nih.gov/books/NBK531485/

4. Wang TB. Diagnosis and treatment of benign multicystic peritoneal mesothelioma. World J Gastroenterol. 2013;19:6689-92. https://doi.org/10.3748/wjg.v19.i39.6689

5. Levy AD, Arnáiz J, Shaw JC, Sobin LH. Primary peritoneal tumors: imaging features with pathologic correlation. RadioGraphics. 2008;28:583-607. https://doi.org/10.1148/rg.282075175

6. Mehta V, Chowdhary V, Sharma R, Golia Pernicka JS. Imaging appearance of benign multicystic peritoneal mesothelioma: a case report and review of the literature. Clin Imaging. 2017;42:133-7. https://doi.org/10.1016/j.clinimag.2016.10.008

7. Özgen A, Akata D, Akhan O, Tez M, Gedikoglu G, Özmen MN. Giant benign cystic peritoneal mesothelioma: US, CT, and MRI findings. Abdom Imaging. 1998;23:502-4. https://doi.org/10.1007/s002619900387
8. Ramirez Y, Rosai J, Segura JJ. Loose Mesothelial cysts in the peritoneal cavity. Inter J Surg Pathol. 2010;18:2146. https://doi.org/10.1177/1066896910362081

9. Torres-Cepeda D, Santos-Bolívar J, Reyna-Villasmil E. Mesotelioma peritoneal quístico benigno. Reporte de caso. Rev Peru Ginecol Obstet. 2016;62:251-5. Disponible en http://www.scielo.org.pe/scielo.php?scrip$\mathrm{t}=$ sci_arttext\&pid=S2304-51322016000200013

10. Gussago S, Spina P, Guerra A. Benign Multicystic Peritoneal Mesothelioma (BMPM) as a rare cause of abdominal pain in a young male: case report and review of the literature. J Surg Case Rep. 2019;19:1-4. https://doi.org/10.1093/jscr/rjz057

11. Cha KS, Choi YH, Lee YS, Park EK. Benign multicystic peritoneal mesothelioma treated with laparoendoscopic single site surgery: a case report and review of the literature. Obstet Gynecol Sci. 2018;61:170-4. https://doi.org/10.5468/ogs.2018.61.1.170

12. García-Mayor RL, Fernández-González M, López-Rodríguez A, Martínez-Almeida R. Mesotelioma multiquísticos peritoneal benigno recidivante: abordaje de esta entidad tan poco frecuente. Cir Cir. 2016;84:499-502. https://doi.org/10.1016/j.circir.2015.08.013

13. Letterie GS, Yon JL. The antiestrogen tamoxifen in the treatment of recurrent benign cystic mesothelioma. Gynecol Oncol. 1998;70:131-3. https://doi.org/10.1006/gyno.1998.5031 